\title{
Commentary
}

\section{SARS-CoV-2 Variants of Concern and Changing Trends of COVID-19 in India and Rajasthan: Epidemiological Perspective}

\author{
Rajeev Gupta ${ }^{1,2}$, Kiran Gaur ${ }^{3}$, Vishwa Mohan Katoch ${ }^{4}$ \\ ${ }^{I}$ Chair, Academic Research Development Unit, Rajasthan University of Health Sciences, ${ }^{2}$ Department of Medicine, \\ Eternal Heart Care Centre and Research Institute; ${ }^{3}$ Department of Statistics, Mathematics and Computer Science, \\ Government SKN Agriculture College, Jobner; ${ }^{4} I C M R-N A S I$ Chair Public Health Research, Rajasthan University of \\ Health Sciences, Jaipur, Rajasthan, India \\ DOI:10.37821/ruhsjhs.6.3.2021.397
}

\section{BACKGROUND}

Fluctuating course of COVID-19 epidemic in India, especially the massive second wave of cases and deaths in multiple states of the country, has attracted worldwide attention. Stringent lockdown along with strict public health mandates regarding universal masking and physical distancing at the beginning of the epidemic in March 2020 led to curtailment of disease spread. ${ }^{1}$ The number of cases and deaths were low in early months of the epidemic but as the lockdown and public health mandates were weaned due to various reasons, a rapid increase was observed which peaked in September-October 2020. ${ }^{1}$ Then, without any explicable reason or changes in the public health mandates, the number of cases as well as deaths started to decline and nadired by January 2020. All this while political, social, and religious events with gatherings of thousands of people and very limited public health mandates continued. ${ }^{1}$ The infection resurfaced with exponential increase in morbidity and mortality in middle of this year. ${ }^{2}$ A number of factors including political, religious, and social gatherings, despite intensive vaccination drive, have been held responsible. ${ }^{3}$ Individual level relaxation of public health mandates could also be important, although there are no large studies in India that assessed public health behavior and adherence to mandates. A novel perspective to explain the fluctuating course of the epidemic in India is biological changes in SARS-CoV-2 virus due to ongoing mutation of the virus genome leading to variants of concerns (VOCs). ${ }^{4}$ A number of factors promote mutations. These are prolonged illness especially among the elderly and the immunocompromised, long-term use of immunosuppressants including injudicious use of steroids, widespread use of plasma therapy, etc. ${ }^{5,6}$ In India, such patients and practices are widely prevalent. ${ }^{1,7}$ Data on VOCs in India are now available at INSACOG (Indian Covid-19 Genome Surveillance) ${ }^{8}$

To correlate changing trends in VOC's with COVID-19 cases and deaths in India and Rajasthan, we performed a descriptive study. The study has been conducted using publicly available data on COVID-19 in India ${ }^{9}$ and SARSCoV-2 variants of concern at INSACOG. ${ }^{8}$ We obtained data for India and Rajasthan and clubbed daily data into weekly and monthly numbers beginning March 2020 to the end of August 2021. INSACOG data for India $(n=61602)$ are available from June 2020 to July 2021. Genomic data for Rajasthan $(\mathrm{n}=1640)$ are available from September 2020 to July 2021. Graphs depicting secular trends were made using raw data in MS PowerPoint. Association of Covid-19 waves in India and Rajasthan with changing VOC's were visually estimated.

\section{OBSERVATIONS}

More than 35 million COVID-19 cases and 430,000 deaths have been reported in India from March 2020 to August 2021. ${ }^{9}$ These numbers could be underestimates. ${ }^{10}$ Trends in COVID-19 cases and deaths per million population in India and Rajasthan from March 2020 to August 2021 are shown in figure 1 . There is a bimodal distribution for India with two peaks in cases and deaths- the first wave peaked in August-November 2020 and the second in April-June 2021. In Rajasthan also (Figure 1) there are similar peaks. Country level trends for various variants (sample size, $\mathrm{n}=$ 61602) from June 2020 to August 2021 are shown in figure

2. It is observed that the dominant strains in the first wave in 

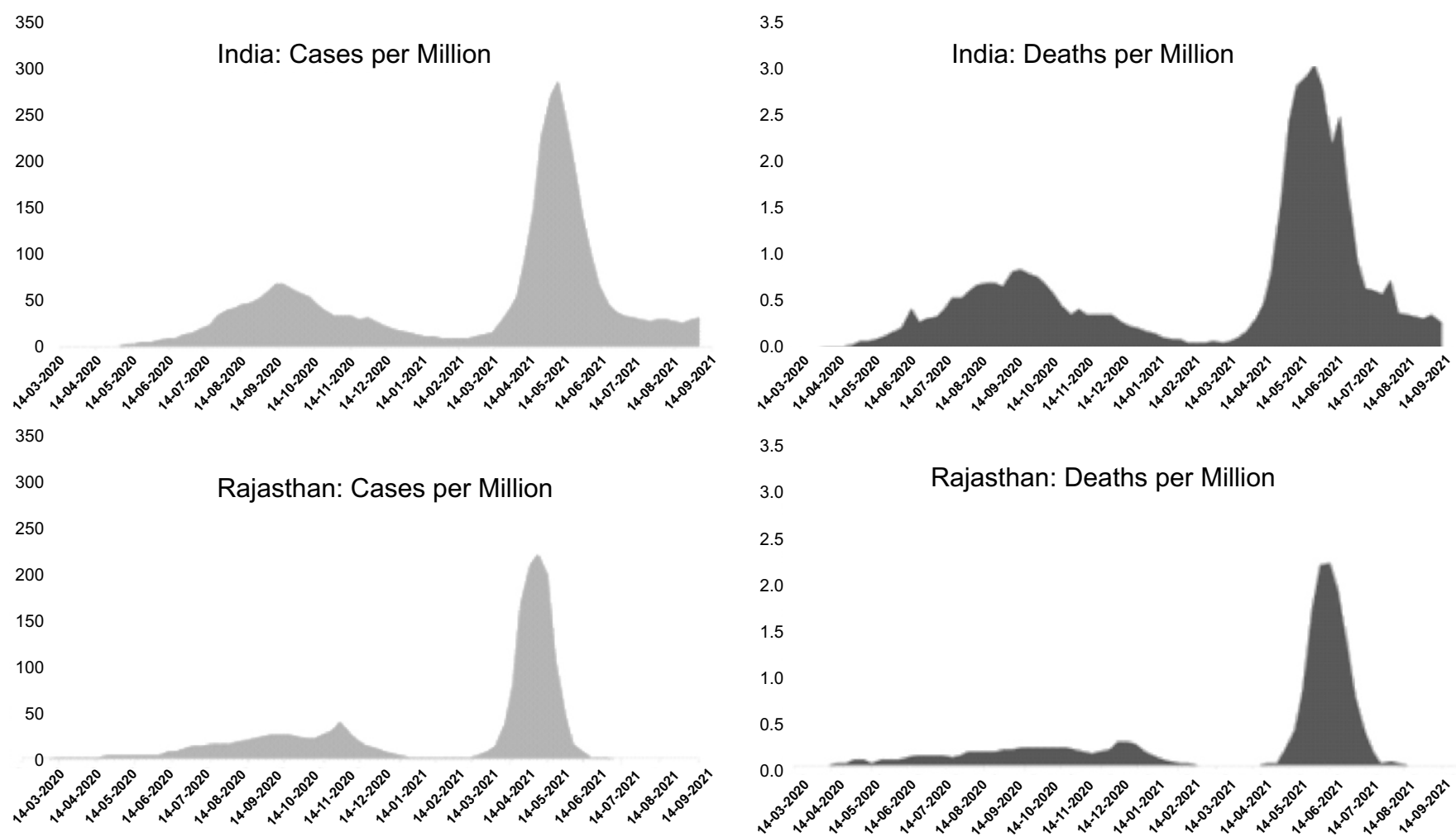

Figure 1: Trends in daily COVID-19 cases and deaths per million population in India and Rajasthan from March 2020 to August 2021.

India were ancestral and other wild types variants along with alpha variants, and in the second wave were predominantly delta variants. In Rajasthan (Figure 3), the first peak correlated with predominantly ancestral variants and the second with delta variants B.1.617.2. Although only a small number of viral genomes have been analysed for India as well as Rajasthan, the trends are significant and compatible with international data. ${ }^{11}$

This report shows that there is a significant change in the proportion of VOC's of SARS-CoV-2 in India over the past 18 months. This correlates with varying trends in COVID19 cases and deaths. The more transmissible VOCsB.1.617.1 and B.1.617.2 are associated with greater burden. Timely information regarding SARS-CoV-2 VOCs is essential to plan evidence-based mitigation measures, targeted vaccine development, and more precise vaccine deployment. ${ }^{12}$ An important limitation is lack of robust COVID-19 epidemiological and mortality data in India and limited availability of sequenced virus genomes. Higher case-burden and mortality in more developed states of the country points to better case-ascertainment due to better healthcare and diagnostic facilities in these states and the database may have missed actual disease burden in lesser developed states of the country. ${ }^{10.13}$ Another limitation is the small proportion of samples that have been processed for genomic analysis and identification of variants, only 60 thousand genomes in more than 35 million COVID-19 patients (0.17\%) have been assessed, while the consensus recommendation is more than $5 \%{ }^{14,15}$ VOC data from some of the larger and most populous states of India, including Rajasthan, are limited. ${ }^{8}$

\section{IMPLICATIONS}

Molecular characterization of the SARS-CoV-2 virus genome has been performed since the beginning of the pandemic and has revealed thousands of variants. ${ }^{4}$ Most of the variation reveals a genetic drift which has helped in tracking the spread of the pandemic and provided important lessons for virus control for public health scientists. Some countries, such as the UK, have been able to successfully analyze almost $10 \%$ of the genomes. ${ }^{15}$ Limited data are available from most of the lower-middle and low-income countries, where the virus has created untold misery. Our study shows that despite low 


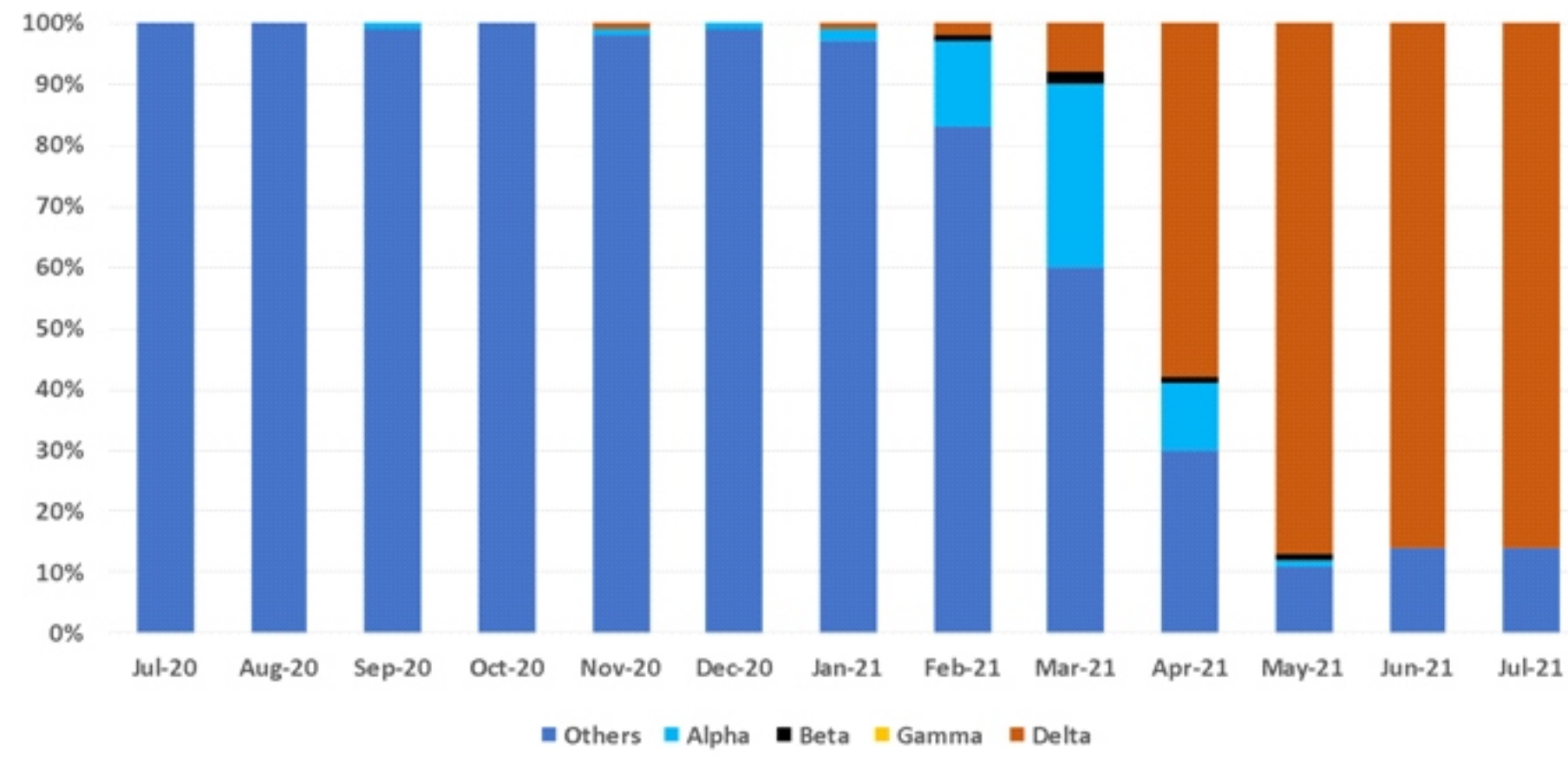

Figure 2: Trends in $S A R S-C o V-2$ variants of concern in India from INSACOG database $(n=61602)$.

availability of the genomic data, trends show significant changes in VOCs that correlate well with the varying trend of pandemic in India and could be useful for prediction of the future waves of the disease. The virus characterization also has helped in rapid vaccine development and immensely effective m-RNA vaccines are a good example. ${ }^{16}$ Minor changes in m-RNA vaccines is possible to prevent variants' induced COVID-19. ${ }^{4}$ Development of a pan coronavirus vaccine is another possibility. ${ }^{17}$

Precision public health has been defined as targeted interventions for better public health.This is an emerging discipline that uses extensive population specific data to provide the right intervention to the right population at the right time. Pathogen genomics has become an important example, especially for COVID-19, and a combination of genome-sequence analyses and epidemiological data have been used for identification of community transmission patterns and rapid deployment of community based interventions. ${ }^{18}$ Similarly, early identification of VOCs can lead to suitable changes in the vaccine ingredients for better efficacy, especially in populations and regions where such variants are evolving or likely to evolve. ${ }^{16}$ For example, In India early identification of B.1.617.2 variant could have resulted in rapid deployment of the 2-dose vaccination program as it is now known that to control this VOC, at least 2 doses of the ChAdOx1 vaccine are required. ${ }^{19}$
Precision public health epidemiology would have been able to guide the policymakers regarding the location of vaccine deployment and suitable inter-vaccine interval. Epidemiological data has shown that low socioeconomic individuals in India are more prone to die of COVID- $19^{20}$, and precision public health policies would have targeted these populations. Identification of strains would also have promoted variant-specific clinical trials to identify the best strategy to curtail the pandemic. Studies in South Africa have focused on B.1.351 strain and found that both ChAdOx1 and NVX-CoV2373 are less effective against this VOC. ${ }^{4}$ Identification of variants shall also help in designing strain-specific or multi-strain vaccines, similar to seasonal influenza vaccines. ${ }^{17}$ Comprehensive SARS$\mathrm{CoV}-2$ genomic analysis also helps to identify potential targets for pharmacotherapy or drug repurposing using artificial intelligence.

In conclusion, the spread of variants of concern of SARSCoV-2 explain the fluctuating course of COVID-19 epidemic in India. Early and rapid identification of the evolving variants is important for guiding precision public health measures to focus on vulnerable populations, nonpharmacological interventions, and vaccine deployment. The identification would also guide vaccine development including the conduct of VOC-specific clinical trials. 


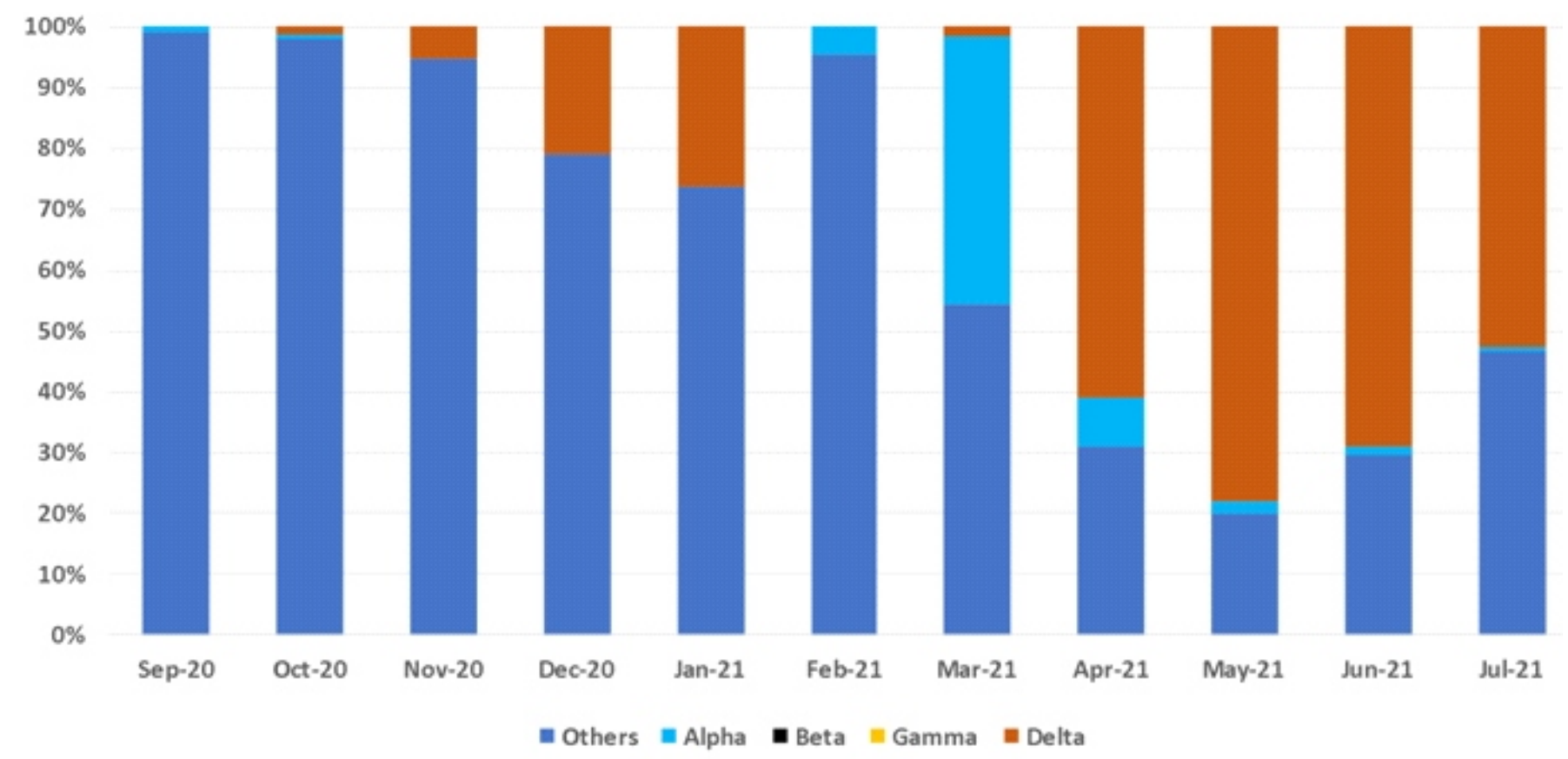

Figure 3: Trends in $S A R S-C o V-2$ variants of concern in Rajasthan from INSACOG database (n=1640).

\section{REFERENCES}

1. Ghosh A. Billions under lockdown: The inside story of India's fight against COVID-19. New Delhi. Bloomsbury India. 2021.

2. Bhuyan A. COVID-19: India sees new spike in cases despite vaccine rollout. BMJ.2021;372:n854.

3. Kuppalli K, Gala P, Cherabuddi K, Kalantri SP, Mohanan M, Mukherjee B et al. India's COVID-19 crisis: A call for international action. Lancet. 2021; 397:2132-35.

4. Kupferschmidt K. New SARS-CoV-2 variants have changed the pandemic: What will the virus do next? Science. 2021. Available at: www.sciencemag.org/news/2021/08/

5. Boehm E, Kronig I, Neher R, , Eckerle I, Vetter P, Kaiser L et al. Novel SARS-CoV-2 variants: The pandemics within the pandemic. Clin Microbiol Infect. 2021; EPub. S1198-743X (21)00262-7.

6. Banoun H. Evolution of SARS-CoV-2: Review of mutations, role of the host immune system. Nephron. 2021; EPub. DOI: 10.1159/000515417.

7. Gupta R. Covid-19 epidemic: Preparing for the next wave. RUHS JHealth Sciences. 2021; 6:79-83.

8. Indian Covid-19 Genome Surveillance, INSACOG. National SARS-CoV-2 genomics sequencing consortium. Available at: http://clingen.igib.res.in/covid19genomes/. Accessed $5^{\text {th }}$ Sep 2021.

9. Anonymous. Coronavirus outbreak in India. Available at:
www.COVID19india.org. Accessed $5^{\text {th }}$ Sep 2021.

10. Deshmukh Y, Suraweera W, Tumbe C, Bhowmick A, Sharma S, Novosad P et al. Excess mortality in India from June 2020 to June 2021 during the Covid pandemic: Death registration, health facility deaths and survey data. medRxiv Preprints. 2021; https://doi.org/10.1101/2021.07.20. 21260872

11. Ong SWX, Chiew CJ, Ang LW, Mak TM, Cui L, Toh MPHS et al. Clinical and virological features of SARS-CoV-2 variants of concern: A retrospective cohort study comparing B.1.1.7 (alpha), B.1.315 (beta) and B.1.617.2 (delta). Clin Infect Dis. 2021; EPub. DOI: 10.1093/cid/ciab721.

12. Chakraborty D, Agrawal A, Maiti S. Rapid identification and tracking of SARS-CoV-2 variants of concern. Lancet. 2021;397:1346-47.

13. The Lancet. India's Covid-19 emergency. Lancet 2021; 397:1683.

14. Center for Disease Control and Prevention. Genomic surveillance for SARS-CoV-2. Available at: https://www. cdc.gov/coronavirus/2019-ncov/variants/variant-surveillance.html?CDC_AA_refVal=https $\% 3 \mathrm{~A} \% 2 \mathrm{~F} \% 2 \mathrm{Fwww} . \mathrm{c}$ dc.gov\%2Fcoronavirus $\% 2 \mathrm{~F} 2019$-ncov\% $2 \mathrm{~F}$ cases-updates \%2Fvariant-surveillance.html. Accessed 31 Aug 2021.

15. Public Health England. National Covid-190 surveillance reports. Available at: https://www.gov.uk/government/ publications/national-covid-19-surveillance-reports. Accessed 2 Sep 2021. 
16. Neuzil KM. Interplay between emerging SARS-CoV-2 variants and pandemic control. $N$ Engl J Med. 2021; 384:1952-54.

17. Cohen J. Vaccines that can protect against many coronaviruses could prevent another pandemic. Science. 2021; EPub May 2021, DOI: 10.1126/science.abi9939.

18. Rasmussen SA, Khoury MJ, del Rio C. Precision public health as a key tool in the COVID-19 response. JAMA.2020; 324:933-34.

19. Wadman M. What does the delta variant have in store for the Unites States? We asked Coronavirus experts. Science. 2021; Available at: https://www.sciencemag.org/news/ 2021/08/what-does-delta-variant-have-store-us-we-askedcoronavirus-experts. Accessed 30 Aug 2021.

20. Sharma AK, Gupta R, BaigVN, Singh TV, Chakraborty S, Sunda JP et al. Socioeconomic status and COVID-19 related outcomes in India: Hospital based study.medRxiv preprints. 2021. https://doi.org/10.1101/2021.05.17.21257364.

\section{Corresponding Author}

Dr Rajeev Gupta, Department of Medicine, Eternal Heart Care Centre and Research Institute, Jagatpura Road, Jawahar Circle, Jaipur, Rajasthan, India

email: rajeevgg@gmail.com; 